\title{
METAIS PESADOS EM ÁGUAS SUPERFICIAIS COMO ESTRATÉGIA DE CARACTERIZAÇÃO DE BACIAS HIDROGRÁFICAS
}

\author{
Maria Josefa Santos Yabe \\ Departamento de Química - Universidade Estadual de Londrina - CP 6001 - 86051-970 - Londrina - PR \\ Elisabeth de Oliveira \\ Instituto de Química - Universidade de São Paulo - CP 26077 - 05599-970 - São Paulo - SP
}

Recebido em 12/2/97; aceito em 29/1/98

\begin{abstract}
HEAVY METALS IN FRESH WATERS FOR EVALUATION OF HYDROGRAPHIC BASINS. The input of heavy metals concentrations determinated by ICP-AES, in samples of the Cambé river basin, was evaluated by using the Principal Component Analysis. The results distinguishes clearly one site, which is strongly influenced by almost all elements studied. Special attention was given to $\mathrm{Pb}$, because of the presence of one battery industry in this area. Some downstream samples were associated with the same characteristics of this site, showing residual action of contaminants along the basin. Other sites presented influence of soil elements, plus $\mathrm{Cr}$ near a tannery industry. This study allowed to distinguish different sites in the upper basin of the Cambé (Londrina-PR$B R$ ), in accordance to elements input.
\end{abstract}

Keywords: heavy metal; fresh water; ICP-AES; chemometrical evaluation.

\section{INTRODUÇÃO}

Os reservatórios naturais vêm sendo depositários de uma variedade de subprodutos, provenientes da atividade antrópica. A presença de elementos potencialmente tóxicos é responsável por efeitos adversos sobre o ambiente, com repercussão na economia e na saúde pública. A introdução de metais nos sistemas aquáticos ocorre naturalmente através de processos geoquímicos, no intemperismo e, a contribuição atribuída à atividade humana é um reflexo de sua ampla utilização pela indústria.

$\mathrm{O}$ crescimento desenfreado de pólos urbanos está alheio às demandas que vão surgindo em função das indústrias instaladas. Estas, por sua vez, desempenham um papel importante na sociedade como geradoras de benefícios, quanto à produção de bens de consumo, impostos e empregos.

Sua implantação em épocas mais remotas, não contava ainda com a aplicação dos relatórios de impacto ambiental (EIA, RIMA). Atualmente, entretanto, exige-se fiscalização dos índices estabelecidos pela legislação ${ }^{1}$ que devem ser cumpridos.

Diversos pesquisadores ${ }^{2-8}$ têm voltado seu interesse para a quantificação de poluição por metais pesados em bacias hidrográficas, reunindo dados sobre o impacto ambiental e suas complexas relações com as atividades econômicas. Os objetivos são de determinação dos níveis e identificação das possíveis fontes de poluição, bem como atribuição de tendências nas mudanças de comportamento na estruturação industrial e na implementação de tecnologias de controle da poluição.

Alguns estudos vêm demonstrando a importância da análise histórica das concentrações de metais pesados em águas superficiais. Esta, possibilita o modelamento de emissões e deposição nas décadas passadas, indo ao encontro de estratégias de controle ${ }^{9-12}$.

Um processo de reversão do estado de deterioração, encontrado em locais específicos de centros urbanos, pode ser instituído por programas de monitoramento e prevenção da degradação da qualidade da água, junto à regulamentação limitante sobre fontes puntuais de descarga.

Com o objetivo de caracterizar a bacia do ribeirão Cambé, Londrina- norte do Pr, quanto contribuição de metais pesados na fração dissolvida, foram realizadas amostragens mensais em oito locais, representativos da bacia com os estudos voltados para pontos de possível interferência antrópica. Foram efetuadas determinações de $\mathrm{Al}, \mathrm{Ca}, \mathrm{Cd}, \mathrm{Cr}, \mathrm{Cu}, \mathrm{Fe}, \mathrm{Mg}, \mathrm{Mn}$, $\mathrm{Ni}, \mathrm{Pb}$ e $\mathrm{Zn}$ por espectrometria de emissão atômica acoplada com plasma de argônio induzido (ICP-AES), com a vantagem da determinação multielementar dos elementos em quantidades maiores, menores, traços e ultraços, sem mudanças nos parâmetros experimentais ${ }^{13-17}$.

\section{EXPERIMENTAL}

\subsection{Amostragem}

As amostragens foram feitas em diferentes pontos da bacia do ribeirão Cambé, figura 1, que atravessa a cidade de Londrina, na região norte do estado do Paraná, usando garrafas plásticas de água mineral, segundo recomendação do Instituto Adolfo Lutz ${ }^{18}$.

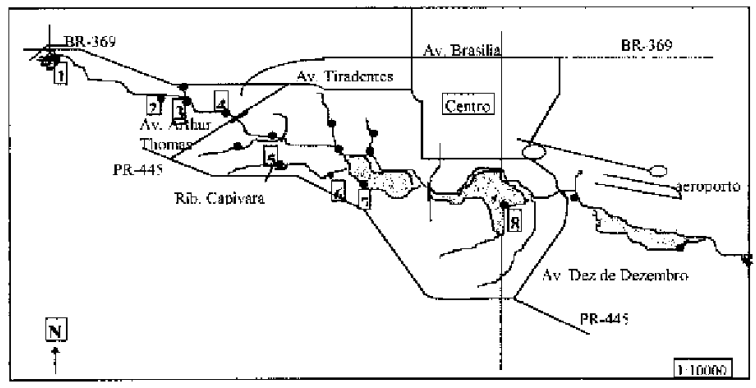

Figura 1. Representação gráfica dos pontos de amostragem de 1 a 8.

Após descartar seu conteúdo, a garrafa foi enxaguada várias vezes com a água amostrada e imersa a $\pm 30 \mathrm{~cm}$, em uma distância aproximada de $2 \mathrm{~m}$ da margem e filtradas com membrana de $0,45 \mu \mathrm{m}^{18}$. Foi realizado um teste para verificar a possibilidade de contaminação das amostras através da membrana de filtração, usando um padrão multilelementar. Os resultados antes e após a passagem do padrão pela membrana não foram significativamente diferentes, 
demonstrando a viabilidade de sua utilização na filtragem das amostras coletadas. As amostras foram filtradas o mais rápido possível, para minimizar a liberação de elementos em baixa concentração do material particulado para a solução, aciduladas ( $1 \mathrm{~mL} \mathrm{HNO}_{3} 1 \% / 100 \mathrm{~mL}$ de amostra) e armazenadas a $4^{\circ} \mathrm{C} \mathrm{em}$ refrigerador. Como a análise deve ser imediata, não devendo ultrapassar as 24 horas após a amostragem ${ }^{19}$, o tratamento das amostras teve início dentro deste prazo. Inicialmente, as amostras foram pré-concentradas 8 vezes por aquecimento convectivo em chapa elétrica, à temperatura aproximada de $60^{\circ} \mathrm{C}$, para assegurar uma concentração metálica suficiente para as determinações, em função do limite de detecção imposto pela técnica de espectrometria de emissão atômica com fonte de excitação de plasma de argônio induzido.

Foi realizado um teste de recuperação para verificar a viabilidade do uso do aquecimento como método de pré-concentração para amostras de água. A adição de padrão, em triplicata, apresentou um índice médio de recuperação de $97 \pm 4 \%$ para os metais estudados, evidenciando a viabilidade do uso do sistema de pré-concentração proposto.

\subsection{INSTRUMENTAÇÃO}

Para a determinação das concentrações metálicas, foi utilizado um ICP-AES sequiencial Spectroflame da Spectro. As condições de operação constam na tabela 1 . As condições ótimas para determinação multielementar foram estabelecidas conforme recomendação do fabricante, para o elemento $\mathrm{Mn}$, canal de alinhamento do espectrômetro.

Tabela 1. Condições de operação do sistema.

$\begin{array}{ll}\text { Gás refrigerante (Ar) } & 8,0 \mathrm{~L} \cdot \mathrm{min}^{-1} \\ \text { gás auxiliar (Ar) } & 8,0 \mathrm{~L} \cdot \mathrm{min}^{-1} \\ \text { pressão do nebulizador } & 2,4 \mathrm{bar} \\ \text { altura de observação vertical } & 12 \mathrm{~mm}^{\mathrm{a}} \\ \text { potência do plasma } & 1,13 \mathrm{Kw} \\ \text { velocidade de introdução da amostra } & 8,0 \mathrm{~L} \cdot \mathrm{min}^{-1}\end{array}$

${ }^{a}$ acima da bobina de indução.

As soluções estoque foram preparadas com metais de pureza elevada (Johnson Matthey), água deionizada (deionizador Permution) e $\mathrm{HNO}_{3}$ grau analítico, em concentração de 1000 $\mu \mathrm{g} . \mathrm{mL}^{-1}$. Soluções de trabalho multielementares foram preparadas em concentrações de 0,$01 ; 0,1 ; 0,5 ; 1 ; 5 ; 10$ e $50 \mu \mathrm{g} . \mathrm{mL}^{-1}$ em $\mathrm{HNO}_{3} 1 \%$ de cada elemento constituinte, por diluição das soluções estoque, e acondicionadas em frascos de polietileno prélavados com $\mathrm{HNO}_{3} 10 \%$. Um branco foi preparado com $\mathrm{HNO}_{3}$ $1 \%$ em água deionizada e estocado da mesma forma.

Interferências espectrais têm sido motivo de preocupação em termos de erro analítico na espectrometria de emissão atômica. A escolha sensata de linhas livres de interferências permite análises mais exatas, precisas e rápidas na determinação de ele mentos. Os comprimentos de onda dos 11 elementos (tabela 2), foram escolhidos com a ajuda de estudos realizados por Garbarino \& Taylor $^{2}$, além da avaliação das linhas livres de interferentes para os elementos em estudo. Como as concentrações metálicas nas amostras estão na faixa de traços $\left(<10 \mu \mathrm{g} \cdot \mathrm{mL}^{-1}\right)$, a maior concentração escolhida para cada elemento da solução de trabalho foi de $10 \mu \mathrm{g} . \mathrm{mL}^{-1}$, com exceção de $\mathrm{Ca}$ e $\mathrm{Mg}$, de 200 $\mu \mathrm{g} \cdot \mathrm{mL}^{-1}$. As concentrações de $\mathrm{Ca}$ e $\mathrm{Mg}$ presentes na amostra possibilitaram a leitura direta, sem pré-concentração.

Assim, para cada elemento foram traçados os espectros: de uma solução de trabalho contendo apenas o analito, da solução multielementar e de uma amostra coletada. De forma geral, não foram verificados problemas de aumento de radiação de fundo ou interferência espectral entre os elementos estudados, para os comprimentos de onda escolhidos. Não foram verificadas interferências de $\mathrm{Na}$ e K, mesmo em concentrações 25 vezes mais elevadas que os demais elementos. Estes não emitem intensamente dentro do intervalo de 1 utilizado nas determinações. As curvas analíticas definidas para cada elemento, mostraram-se essencialmente lineares a partir do limite de detecção até a concentração mais elevada (tabela 2). As curvas analíticas de $\mathrm{Cr}$ e $\mathrm{Cu}$ foram traçadas usando polinômio de $2^{\circ}$ grau, e somente para o $\mathrm{Pb}$ foi corrigida a radiação de fundo. Os dados obtidos ofereceram os subsídios necessários ao diagnóstico pretendido nos objetivos iniciais do trabalho.

Tabela 2. Comprimentos de onda, parâmetros da curva analítica e limites de detecção para cada elemento.

\begin{tabular}{lccccc}
\hline $\mathrm{M}$ & $\begin{array}{c}\lambda \\
(\mathrm{nm})\end{array}$ & $\begin{array}{c}\text { L.D. } \\
(\mu \mathrm{g} / \mathrm{L})\end{array}$ & $\mathrm{A}_{0}$ & $\mathrm{~A}_{1}$ & $\mathrm{r}$ \\
\hline $\mathrm{Cd}$ & 214,421 & 3,3 & $-0,143$ & 0,958 & 1,0000 \\
$\mathrm{Ni}$ & 232,002 & 9,7 & $-0,250$ & 1,870 & 0,9996 \\
$\mathrm{Mn}$ & 257,612 & 0,8 & $-0,033$ & 0,071 & 0,9996 \\
$\mathrm{Fe}$ & 259,941 & 3,4 & $-0,075$ & 0,306 & 0,9999 \\
$\mathrm{Cr}$ & 267,715 & 5,6 & $-0,132$ & 0,715 & 0,9988 \\
$\mathrm{~Pb}$ & 220,349 & 2,1 & $-0,116$ & 7,410 & 0,9996 \\
$\mathrm{Mg}$ & 285,208 & 1,3 & $-0,044$ & 0,083 & 0,9999 \\
$\mathrm{Ca}$ & 317,939 & 11 & $-0,300$ & 0,353 & 0,9999 \\
$\mathrm{Cu}$ & 324,743 & 2,8 & $-0,100$ & 0,067 & 0,9993 \\
$\mathrm{Zn}$ & 213,844 & 2,2 & $-0,090$ & 0,560 & 1,0000 \\
$\mathrm{Al}$ & 396,146 & 14 & $-0,33$ & 285, & 0,9999 \\
\hline
\end{tabular}

L.D. - limite de detecção; r- coeficiente de correlação da regressão, $\mathrm{A}_{0}$ - coeficiente linear, $\mathrm{A}_{1}$ - coeficiente angular da regressão.

\section{RESULTADOS E DISCUSSÃO}

Organizar, interpretar e analisar o grande número de dados obtidos devido ao caráter do trabalho, bem como extrair informações sobre as principais tendências da variabilidade dos parâmetros: concentração dos diversos metais, $\mathrm{pH}$ e sazonalidade, trouxe a necessidade do uso de uma ferramenta matemática, a estatística multivariada. Esta pode ser aplicada quando é adquirida mais de uma medida de cada amostra, na aquisição de dados múltiplos. Portanto, para identificar as relações entre os dados obtidos, foi utilizada a técnica da análise multivariada ${ }^{20-22}$. $\mathrm{O}$ método das componentes principais pode resumir em poucas e importantes dimensões a maior parte da variabilidade de uma matriz de dados de um grande número de variáveis, sendo que a variância explicada por estes poucos eixos principais independentes pode ser conhecida.

A estação 1 de amostragem, que constitui o manancial da bacia do ribeirão Cambé, apresenta água límpida e transparante. $\mathrm{O}$ propósito da escolha deste local foi o estabelecimento de uma área de referência para relações com as demais estações de amostragem. A figura 2(a) mostra as correlações mais elevadas entre alguns pares de variáveis do manacial. As altas correlações entre os elementos $\mathrm{Pb}, \mathrm{Ni}, \mathrm{Cr}$ e $\mathrm{Cu}$; $\mathrm{Ca}$ e $\mathrm{Mn}$; e $\mathrm{Ca}$ e $\mathrm{Mg}$, mostram interferência do intemperismo, devido às águas rasas, com inúmeras nascentes, que concorrem para o leito do ribeirão. De comportamento ácido, com pH variando entre 3,7 e 5,0, a água do manancial tende a manter os metais em solução. Mesmo com estas correlações, as concentrações metálicas normalmente estiveram abaixo dos valores delineados por órgãos reguladores de concentrações metálicas em águas superficais de classe 2. Segundo a resolução CONAMA 20 de 1986, do Ministério do Desenvolvimento Urbano e Meio Ambiente ${ }^{1}$, as águas de classe 2 são passíveis de tratamento convencional para potabilização e distribuição à comunidade. Com correlação significativa apenas entre o pares de variáveis $\mathrm{Ca}-\mathrm{Mg}$, a figura 2(b) também mostra correlações interessantes entre $\mathrm{Fe}-\mathrm{Al}$ e $\mathrm{Pb}-\mathrm{Cu}$. Aspectos interessantes de correlação entre as variáveis são observados na figura 2(c): ocorrem 
(a)

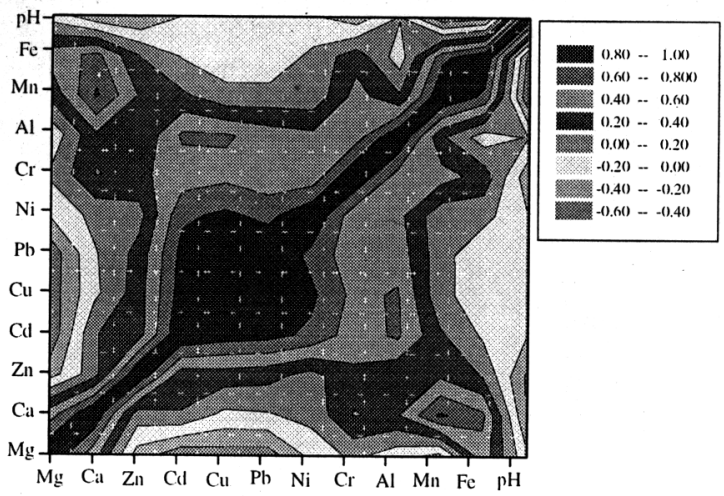

(c)

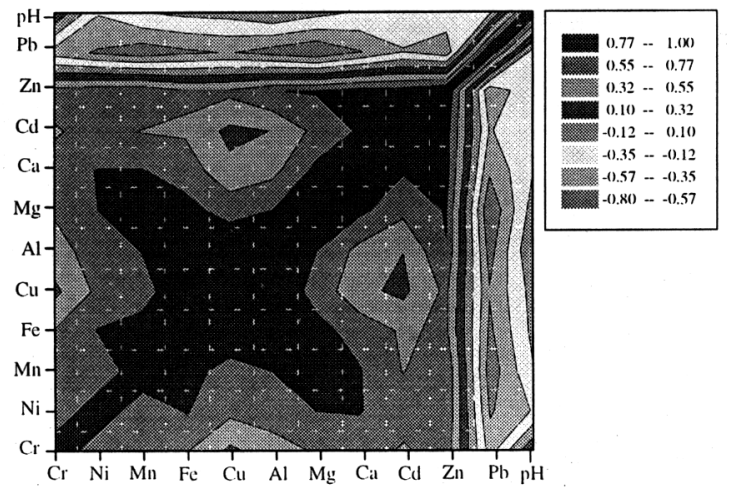

(b)

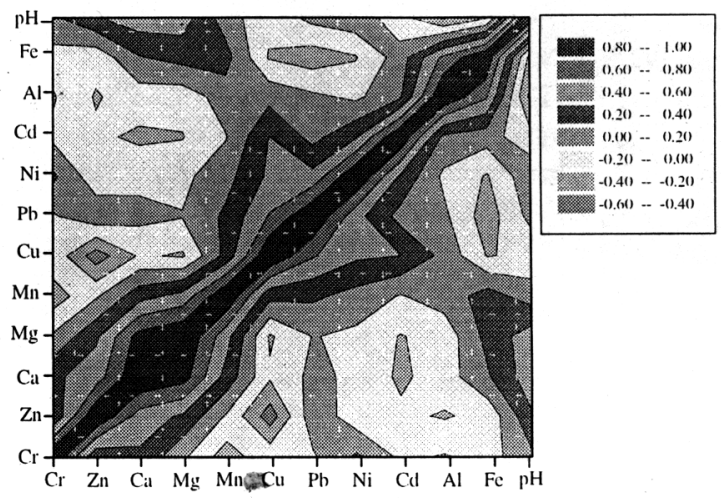

(d)

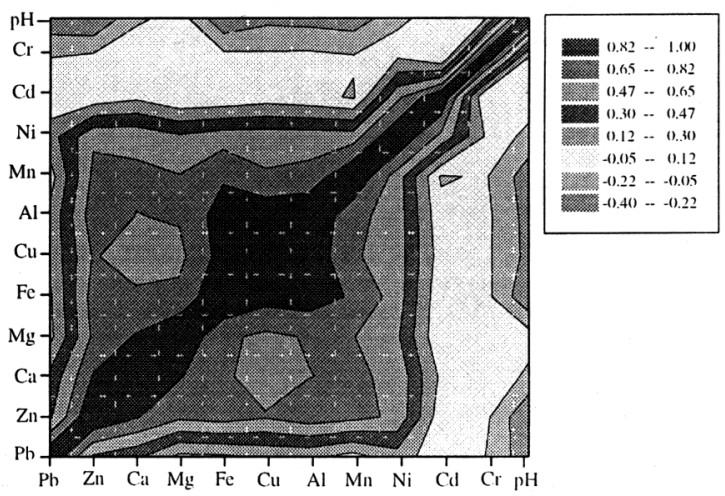

Figura 2. Correlações entre as variáveis das estações de amostragem: (a) manancial, (b) região de um curtume, (c) região de uma fábrica de baterias e (d) bacia completa.

correlações significativas entre quase todos os pares de variáveis, sugerindo a atuação do efluente industrial na introdução de todos os elementos no sistema aquático. $\mathrm{O} \mathrm{Pb}$, correlaciona-se negativamente, em maior ou menor proporção, com todos os outros elementos, definindo um antagonismo deste elemento com os demais. Após a análise individual das estações de amostragem, todos os dados disponíveis foram reunidos em uma só matriz e o estudo de componentes principais respondeu com a nítida separação do local 6. Na figura 2(d) aparecem correlações significativas entre os elementos $\mathrm{Mn}, \mathrm{Al}, \mathrm{Fe}, \mathrm{Cu}, \mathrm{Zn}, \mathrm{Ca}$ e $\mathrm{Mg}$.

As duas primeiras componentes principais, que explicam sozinhas $67,5 \%$ da variância total dos dados, têm contribuições significativas dos elementos que apresentaram as mais altas correlações (figura 2). A configuração em duas dimensões, das duas primeiras componentes principais, dos pontos referentes às amostras, está representada na figura 3. A proximidade das amostras relativas às triplicadas, nas componentes 1 e 2 , demonstra a viabilidade do uso de aquecimento como método de pré-concentração para amostras de água. Os pequenos quadros esclarecem quanto à influência de determinados elementos sobre as amostras. Os elementos $\mathrm{Pb}, \mathrm{Cd}, \mathrm{Ni}, \mathrm{Cr}$ e $\mathrm{Cu}$ têm influência sobre as amostras que representam as triplicatas dos meses de janeiro e fevereiro, deslocando-as das demais e demonstrando a ação das chuvas no carreamento de partículas para o sistema aquático. São observadas separações dos meses mais quentes e chuvosos pelos elementos $\mathrm{Mn}, \mathrm{Fe}, \mathrm{Ca}$ e $\mathrm{Mg}$. O pH interfere principalmente nas amostras de junho e julho. Estes foram os de maior estiagem, com valores de $\mathrm{pH}$ mais elevados. As amostras distribuídas mais à direita, na figura 3(a) sofrem maior influência de Fe. Elementos característicos de solo: $\mathrm{Mn}, \mathrm{Ca}$ e $\mathrm{Mg}$ discriminam as amostras referentes aos meses de maior pluviosidade. Normalmente, as amostras que sofreram maior ação das chuvas, com carreamento de grande quantidade de partículas para o sistema aquático, agrupam-se separadamente das que pertencem à época de seca. Considerando a maior proporção da informação estatística dos dados para as duas primeiras componentes, na figura 2(b), são discriminadas as amostras do local 2, a montante do curtume, daquelas que sofrem a intervenção do efluente no local 3 e no 4, posterior ao despejo. Os três pontos separados no gráfico, mais abaixo, por interferência de $\mathrm{Pb}, \mathrm{Ni}$ e $\mathrm{Cu}$ referemse às amostras de janeiro, em cada estação de amostragem. As amostras restantes são discriminadas por $\mathrm{Zn}, \mathrm{Ca}, \mathrm{Mg}$ e pH. As amostras dos locais 3 e 4, guardam maior semelhança entre si.

Nos meses mais frios e secos, na figura 3(c), os elementos que influenciaram na separação foram $\mathrm{Cu}$ e $\mathrm{Al}$. O aumento de $\mathrm{pH}$ nas amostras de agosto separaram-nas das demais. Setembro e novembro receberam a influência de todos os elementos. As amostras mais à direita na figura sofrem a influência do $\mathrm{Pb}$. Mesmo com o peso pouco abaixo do valor de corte escolhido para os pesos das variáveis, este elemento tem influência marcante sobre a distinção da maioria das amostras. Ao longo do ano, as concentrações de $\mathrm{Pb}$ extrapolam em grande proporção os níveis máximos permitidos pela legislação. Metal tóxico mais abundante, pode ser encontrado em praticamente todas as fases do ambiente e em todos os sistemas biológicos.

Numerando as estações de amostragem de 1 a 8 , a figura 3(d) define bem a discriminação do local 6 , região da fábrica de baterias, cujas amostras sofrem maior influência dos elementos incluídos na composição da primeira componente. A maioria das variáveis estudadas estão incluídas nas duas primeiras componentes, colocando como fator determinante a interferência das mesmas, na discriminação deste local com relação ao restante da bacia. $\mathrm{O} \mathrm{Pb}$, o $\mathrm{Cr}$ e o $\mathrm{pH}$, são os principais agentes discriminadores destas amostras, separando-as das demais.

A similaridade das amostras, estudada pela análise de grupos, auxilia na interpretação dos dados. Quando a matriz de dados é transposta, a análise de grupos pode oferecer subsídios para a compreensão de possíveis fontes dos elementos metálicos no sis- 
a)

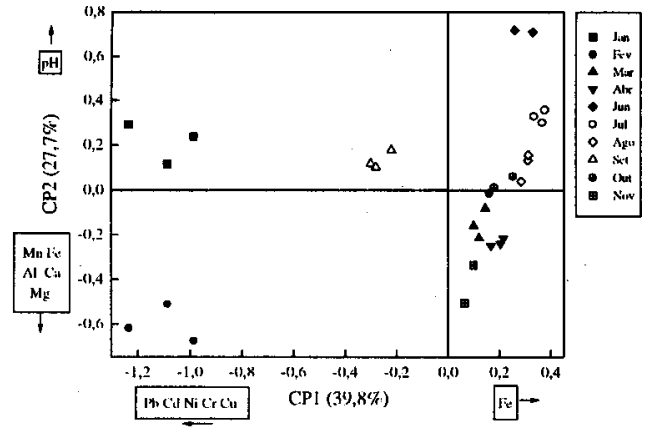

b)

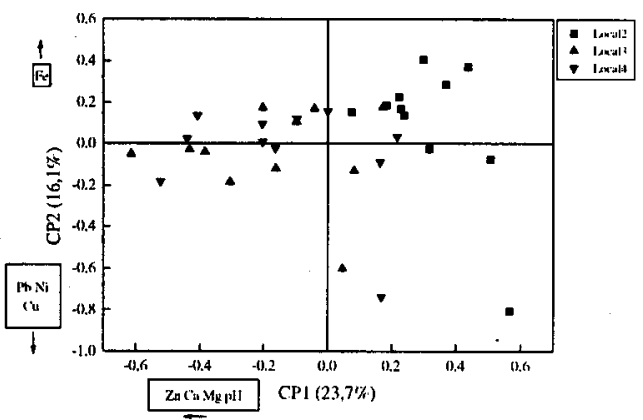

(c)

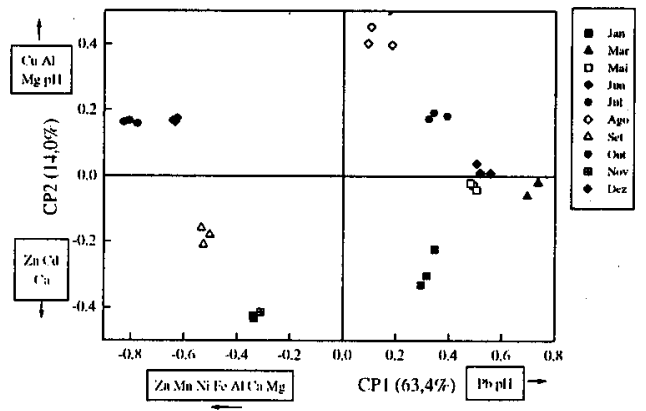

d)

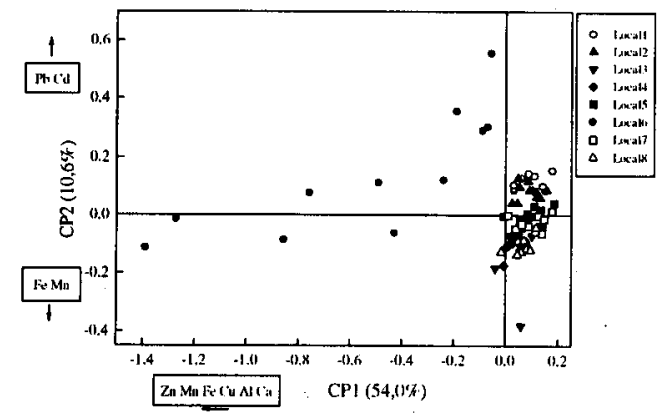

Figura 3. Gráfico das componentes principais 1 e 2 das estações de amostragem: (a) manancial, (b) região de um curtume, (c) região de uma fábrica de baterias e (d) bacia completa. A variância explicada pelas componentes 1 e 2 está discriminadas nos eixos.

tema aquático. $\mathrm{Na}$ figura 4 , os elementos $\mathrm{Pb}, \mathrm{Ni}, \mathrm{Cr}, \mathrm{Cd}$ e $\mathrm{Cu}$, de elevado grau de correlação, agrupam-se com similaridade máxima, indicando a mesma proveniência ao longo da bacia. Elementos de origem mais provável no solo como $\mathrm{Zn}, \mathrm{Al}, \mathrm{Mn}, \mathrm{Fe}$ e $\mathrm{Mg}$ distinguem-se em outro grupo. $\mathrm{O} \mathrm{Ca}$ e o $\mathrm{pH}$ associam-se às demais variáveis, num grau de similaridade menor.Uma grande semelhança nas fontes de metais no início da bacia do ribeirão Cambé, coloca em destaque a região da fábrica de baterias. Em função da resposta de correlação elevada entre a maioria dos elementos estudados, o estudo de similaridade auxilia na identificação da fábrica como mais provável fonte de metais como $\mathrm{Cd}, \mathrm{Ni}$,
$\mathrm{Cr}, \mathrm{Cu}, \mathrm{Mn}$ e $\mathrm{Al}$, associados com alto grau de similaridade ainda a $\mathrm{Mg}, \mathrm{Pb}$ e $\mathrm{pH}$, sendo este determinante quanto à solubilidade de metais. Já em um local de menor aglomeração urbana a bacia apresenta de forma escalonada a presença dos elementos estudados, sem discriminação mais evidente sobre suas fontes, a não ser por aqueles elementos provenientes do solo.

a)

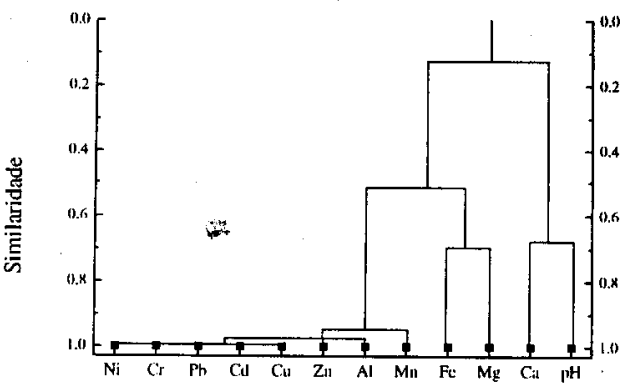

b)

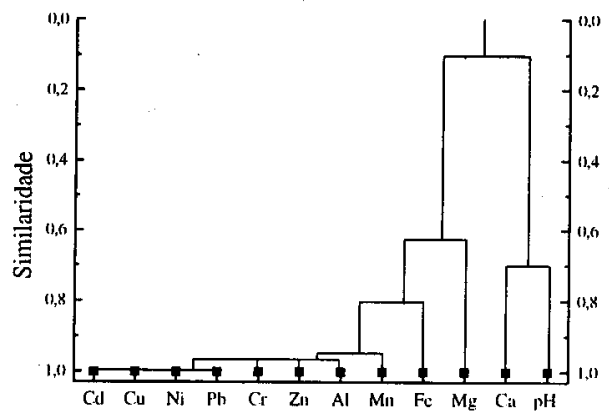

c)

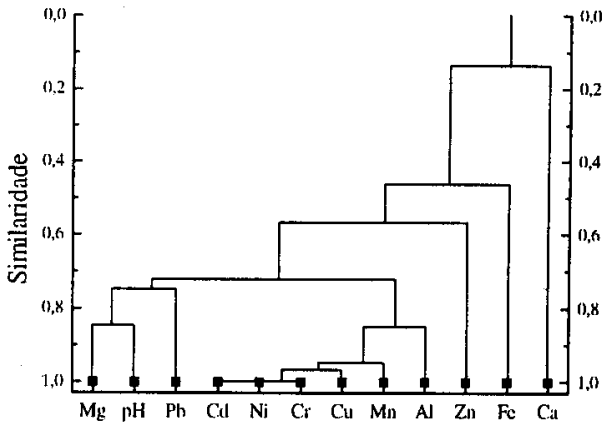

d)

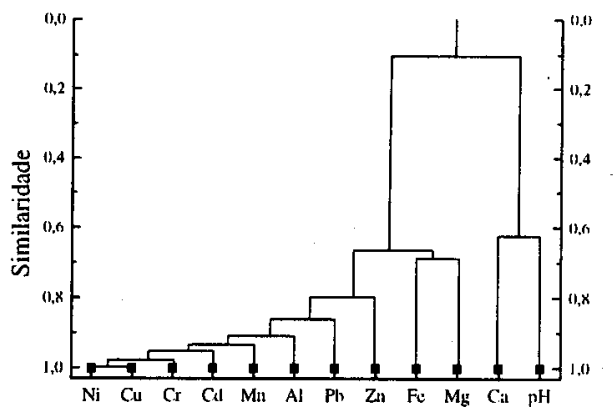

Figura 4. Similaridade entre as variáveis estudadas nas estações de amostragem: (a) manancial, (b) região de um curtume, (c) região de uma fábrica de baterias e (d) bacia completa.

Foram verificados pulsos de concentração (tabela 3) para os elementos que regem as cinco primeiras componentes no manancial e aparecem com as maiores correlações obtidas (figura 2). Em valores de $\mathrm{pH}$ baixos $(<4,5)$, os íons metálicos tendem a permanecer em solução ${ }^{19}$. Aqueles meses nos quais o $\mathrm{pH}$ foi mais alto e a pluviosidade baixa: abril, junho e julho, foram acompanhados pela redução nas concentrações da maioria dos elementos. Com chuvas muito intensas em janeiro, o material depositado por uma eletrosolda, mesmo que distante, pode ter influenciado na contribuição de metais como $\mathrm{Pb}, \mathrm{Cd}$, 
Tabela 3. Concentração média \pm desvio padrão, em $\mu \mathrm{g} / \mathrm{L}$.

\begin{tabular}{|c|c|c|c|c|c|c|}
\hline Metal & Manancial & Curtume & FBmontante $^{\mathrm{a}}$ & FBlocal & FBjusante & Igapó \\
\hline $\mathrm{Pb}$ & $\begin{array}{l}60,12 \\
58,51^{\text {b }}\end{array}$ & $\begin{array}{l}69,01 \\
62,88\end{array}$ & $\begin{array}{l}288,0 \\
221,5\end{array}$ & $\begin{array}{r}4504 \\
418\end{array}$ & $\begin{array}{r}509,9 \\
71,4\end{array}$ & $\begin{array}{l}84,69 \\
33,70\end{array}$ \\
\hline $\begin{array}{l}\mathrm{Zn} \\
\mathrm{Cd}\end{array}$ & $\begin{array}{l}46,20 \\
2,220 \\
0,973\end{array}$ & $\begin{array}{r}52,26 \\
54,20 \\
0,51\end{array}$ & $\begin{array}{l}56,71 \\
3,823 \\
0,263\end{array}$ & $\begin{array}{r}5309 \\
454,1 \\
31,9\end{array}$ & $\begin{array}{l}148,6 \\
4,975 \\
0,675\end{array}$ & $\begin{array}{l}108,3 \\
3,033 \\
2,628\end{array}$ \\
\hline $\mathrm{Mn}$ & $\begin{array}{r}364,7 \\
31,9\end{array}$ & $\begin{array}{l}319,2 \\
124,6\end{array}$ & $\begin{array}{l}367,0 \\
299,3\end{array}$ & $\begin{array}{r}44,76 \\
9,70\end{array}$ & $\begin{array}{l}262,2 \\
207,0\end{array}$ & $\begin{array}{l}67,83 \\
6,425\end{array}$ \\
\hline $\mathrm{Ni}$ & $\begin{array}{l}27,10 \\
18,67\end{array}$ & $\begin{array}{l}27,22 \\
16,69\end{array}$ & $\begin{array}{l}318,0 \\
270,4\end{array}$ & $\begin{array}{r}57,09 \\
4,40\end{array}$ & $\begin{array}{r}51,64 \\
6,48\end{array}$ & $\begin{array}{l}52,58 \\
5,535\end{array}$ \\
\hline $\mathrm{Cr}$ & $\begin{array}{l}30,10 \\
29,07\end{array}$ & $\begin{array}{l}35,06 \\
26,98\end{array}$ & $\begin{array}{l}8,933 \\
0,303\end{array}$ & $\begin{array}{l}6,115 \\
3,280\end{array}$ & $\begin{array}{l}8,180 \\
0,270\end{array}$ & $\begin{array}{r}5,920 \\
2,17\end{array}$ \\
\hline $\mathrm{Fe}$ & $\begin{array}{l}2733 \\
2689\end{array}$ & $\begin{array}{l}777,7 \\
702,3\end{array}$ & $\begin{array}{l}577,8 \\
524,1\end{array}$ & $\begin{array}{l}338,1 \\
212,0\end{array}$ & $\begin{array}{l}226,0 \\
154,9\end{array}$ & $\begin{array}{l}63,36 \\
37,77\end{array}$ \\
\hline $\mathrm{Cu}$ & $\begin{array}{r}1,550 \\
-\end{array}$ & $\begin{array}{l}33,42 \\
29,58\end{array}$ & $\begin{array}{r}10,40 \\
2,83\end{array}$ & $\begin{array}{l}22,40 \\
10,53\end{array}$ & $\begin{array}{l}35,16 \\
30,16\end{array}$ & $\begin{array}{l}7,93 \\
4,23\end{array}$ \\
\hline $\mathrm{Al}$ & $\begin{array}{l}61,17 \\
21,71\end{array}$ & $\begin{array}{r}163,9 \\
31,4\end{array}$ & $\begin{array}{l}281,9 \\
174,2\end{array}$ & $\begin{array}{l}461,4 \\
242,3\end{array}$ & $\begin{array}{r}209,6 \\
89,6\end{array}$ & $\begin{array}{l}96,38 \\
12,88\end{array}$ \\
\hline $\mathrm{Ca}$ & $\begin{array}{r}5049 \\
146\end{array}$ & $\begin{array}{r}5066 \\
88\end{array}$ & $\begin{array}{r}5254 \\
413\end{array}$ & $\begin{array}{r}10969 \\
981\end{array}$ & $\begin{array}{r}6476 \\
811\end{array}$ & $\begin{array}{r}9294 \\
606\end{array}$ \\
\hline $\mathrm{Mg}$ & $\begin{array}{r}2168 \\
426\end{array}$ & $\begin{array}{r}1747 \\
83\end{array}$ & $\begin{array}{r}1348 \\
179\end{array}$ & $\begin{array}{r}1816 \\
205\end{array}$ & $\begin{array}{r}1738 \\
204\end{array}$ & $\begin{array}{r}3801 \\
641\end{array}$ \\
\hline
\end{tabular}

${ }^{\mathrm{a}}$ Fábrica de baterias; ${ }^{\mathrm{b}}$ desvio-padrão

$\mathrm{Ni}, \mathrm{Cu}$ e $\mathrm{Zn}$ em proporções diferentes com relação às demais amostras, provocando uma anomalia naquela àrea.

Por um lado existe a incorporação de metais estranhos ao corpo d'água, provenientes da atividade antrópica, e por outro a contribuição de elementos participantes dos ciclos hidrogeoquímicos. A precipitação de colóides formados devido a variações pequenas de $\mathrm{pH}$ pode provocar processos de arraste por oclusão ou adsorção dos mais diferentes íons. A precipitação de oxihidróxido de Fe ocorre a partir de $\mathrm{pH}$ 2,2. A medida que o $\mathrm{pH}$ aumenta nas amostras analisadas, são verificadas quedas nas concentrações de $\mathrm{Fe}$, bem como da maioria dos metais estudados. Mesmo com as concentrações abaixo do permitido, a variação no mês de agosto deve ter ocorrido devido a uma queda pequena no $\mathrm{pH}$, aumentando a solubilidade dos íons metálicos. Este local está inserido em uma área pastoril, próxima a um pontilhão de autoestrada. As contribuições metálicas associadas a esta estação de amostragem, podem estar relacionadas ao acúmulo de partículas no solo ao longo do tempo, decorrente do uso da autoestrada que conta com tráfego intenso. Com as chuvas fortes, é consequente a introdução de partículas estranhas ao sistema aquático. Mas, considerando que normalmente os níveis obtidos estão dentro dos padrões da legislação, e ainda, acompanhando a sazonalidade, podemos tomar esta área como referência para efeito de comparação com as demais estudadas.

Áreas de referência apresentam um comportamento próprio, nas quais as características estão relacionadas à menor perturbação sobre sua homeostase. Em função das observações, é pertinente inferir quanto à perturbação do sistema. Esta é pressuposta por ações naturais, permitindo considerá-la área de referência, para efeito de comparação com os demais locais estudados. A jusante, houve um aumento na maioria das concentrações, associado às características da próxima estação de amostragem. Trata-se de um curtume, cujo sistema de tratamento de efluente conta com duas lagoas de estabilização. Em despejos ricos em crômio, segundo Eckenfelder ${ }^{24}$, o tratamento envolve um processo de redução de $\mathrm{Cr}^{6+} \mathrm{a} \mathrm{Cr}^{3+}$ com posterior precipitação com cal. Os agentes comumente usados para a redução são sulfato ferroso, metabissulfito de sódio ou dióxido de enxofre. Maior efetividade na redução é atingida em $\mathrm{pH}$ ácido, abaixo de 3,0. Como o pH das amostras estudadas apresentou uma faixa de variação de 7,0-7,8, as concentrações de $\mathrm{Fe}$ mantiveram-se como nos locais a montante e as de $\mathrm{Ca}$ foram praticamente dobradas, evidenciando-se o tratamento do efluente. Ocorre um aumento significativo na concentração de $\mathrm{Cr}$ em fevereiro, março e junho, e menor variação, mas significativa, em agosto e setembro, no local 3 , excedendo em grande proporção os níveis determinados tanto no local 1, referência, como no 2. As maiores correlações entre $\mathrm{Ca}, \mathrm{Mg}, \mathrm{Zn}, \mathrm{Cu}$, $\mathrm{Pb}$ e $\mathrm{Al}$, e menores, mas de importância na avaliação deste local, envolvendo $\mathrm{Cr}$ e outros elementos, estabelecem as diferenças desta estação com relação às duas a montante, caracterizando-a quanto à sua vulnerabilidade. Na região próxima à fábrica de baterias, ocorreram variações bruscas de $\mathrm{pH}$, com aumento significativo nas concentrações metálicas. Estas estiveram em sua grande maioria, acima dos limites máximos permitidos pela lei. Em junho e novembro, cujas amostras apresentaram os menores índices de $\mathrm{pH}$, ocorreram picos na maioria das concentrações. Aquelas amostras cujos valores de $\mathrm{pH}$ foram mais elevados, apresentaram as menores concentrações. Já setembro, com pH também elevado, para esta localidade, aparece com concentrações mais altas, provavelmente em função das chuvas. Confirma-se, então, a hipótese inicial de contaminação na área delimitada pela instalação da fábrica de baterias.

\section{CONCLUSÕES}

Devido às concentrações terem sofrido um aumento generalizado ao longo da bacia, quando comparadas à primeira estação de amostragem, usada como referência, é interessante analisar sua evolução durante o ano. Durante todo o percurso amostrado do ribeirão, houve a presença sistemática dos elementos $\mathrm{Pb}, \mathrm{Ni}, \mathrm{Cd}, \mathrm{Cr}$ e $\mathrm{Cu}$, introduzidos por fontes, discriminadas através da análise de grupos, cuja origem está na urbanização e industrialização, e Fe, $\mathrm{Al}, \mathrm{Mn}, \mathrm{Ca}$ e $\mathrm{Mg}$, como produto, principalmente, do carreamento de partículas, através das águas de escoamento. Meses de maior pluviosidade 
apresentaram aumento nas concentrações metálicas, provavelmente, em função das características do solo e carreamento intenso de partículas para o sistema aquático. Relações entre sazonalidade, $\mathrm{pH}$ e concentração metálica, de forma geral, demonstraram a influência de elementos como Fe e Al em valores de $\mathrm{pH}$ menores, provenientes de processos hidrogeoquímicos ${ }^{25-29}$, acompanhados de aumento nos níveis de $\mathrm{Pb}$, $\mathrm{Cd}, \mathrm{Ni}, \mathrm{Cr}, \mathrm{Cu}$ e $\mathrm{Mn}$, que participaram com correlações significativas ao longo da bacia.

A comparação entre a área de referência e as demais estações de amostragem estabeleceu diferenças positivas, dentro de uma faixa, nas concentrações metálicas, para todas as estações estudadas. Deve ser dada maior atenção ao local 6, como área crítica de contaminação, devido às proporções extraordinárias nas diferenças com relação não só à referência, como à microbacia de forma geral. Como ponto crítico, exige cuidados no que se relaciona ao planejamento, quanto às necessidades de controle na emissão de metais pesados e monitoramento.

\section{REFERÊNCIAS}

1. Ministério do Desenvolvimento Urbano e Meio Ambiente 1986, Resolução ${ }^{\circ}$. 20 de 18 de Junho de 1986. D. O. U. Executivo, 11356.

2. Garbarino, J. R.; Taylor, H. E.; An Inductive-couplesd Plasma. Spectroc. 1979, 33, 220.

3. Bodo, B. A.; Sci. Tot. Environ. 1989, 87/88, 329.

4. Förstner, U.; Schoer, J.; Knauth, H-D.; Sci. Tot. Environ. 1990, 97/98, 347.

5. Taylor, H. E.; Garbarino, J. R.; Brinton, T. I.; Sci. Tot. Environ. 1990, 97/98, 369.

6. Cossa, D.; Tremblay, G. H.; Gobeil, C.; Sci. Tot. Environ. 1990, 97/98, 369.

7. Bubb, J. M.; Lester, J. N.; Sci. Tot. Environ. 1991, 100, 207.

8. Ihnat, M., Gamble, D. S.; GILchrist, G. F. R.; Intern. J. Environ. Anal. Chem. 1993, 53, 63.

9. Hungspreugs, M.; Dharmvanji, S.; Utoomprukpom, W.; Sci. Tot. Environ. 1990, 97/98, 89.

10. Windom, H. L.; Byrd, J. T.; Smith, R. G. Jr.; Huan, F.; Environ. Sci. Technol. 1991, 25, 1137.

11. Windom, H. L.; Byrd, J. T.; Smith, R. G. Jr.; Huan, F.; Environ. Sci. Technol. 1991, 25, 1940.
12. Stigliani, W. M.; Jaffé, P. R.; Anderberg, S.; Environ. Sci. Technol. 1993, 27, 786.

13. Robin, J.; The Determination of Traces Metals in Natural Waters; IUPAC, West, T. S. \& Nürnberg, H. W., London, 1988, 362p.

14. Betti, M.; Pappof, P.; CRC Anal. Chem. 1988, 19, 271.

15. Gabarino, J. R.; Taylor, H. E.; Appl. Spectroc. 1979, 33, 220.

16. Winge, R. K.; Fassel, V. A.; Peterson, V. J.; Floyd, M. A.; Appl. Spectrosc. 1982, 36, 210.

17. Winge, R. K.; Peterson, V. J.; Fassel, V. A.; Appl. Spectrosc. 1979, 33, 206.

18. Normas Analíticas do Instituto Adolfo Lutz. 1985, Adolfo Lutz, São Paulo, 1, 533p.

19. Florence, T. M.; Batley, G. E.; CRC in Anal. Chem. 1980, 9, 219.

20. Scarmínio, I.; Tese de Doutorado. 1989, Universidade Estadual de Campinas, Campinas, SP.

21. Legendre, L.; Legendre, P.; Numerical Ecology. 1983, Elsevier, NY, 419p.

22. Beebe, K. R.; and Kowalski, B. R.; Anal. Chem. 1987, 59, 1007A.

23. Cassaret, L. J.; and Doull, J.; Toxicology 1991, Pergamon Press, NY, p.623.

24. Eckenfelder Jr.; W. W. Industrial Water Pollution Control 1989, McGrawl-Hill, 2ed., 400p.

25. Berman, S.; and MacLaren, J. W.; Appl. Spectrosc. 1978, $32,372$.

26. Westall, J.; and Stumm, W.; The Handbook of Environmental Chemistry 1986, Springer-Verlag, NY, 1, part A 2ed., p. 17.

27. Stumm, W.; and Morgan, J. J.; Aquatic Chemistry, 1981, Wiley-Interscience, NY, 583p.

28. Stumm, W.; Chemistry of the Solid-Water Interface. 1992, John-Wiley \& Sons, Inc., NY, 428p.

29. Bird, C.; Environmental Chemistry 1995, Freeman, NY, 484p.

30. Fairman, B.; and Sanz-Medel.; Int. J. Environ. Anal. Chem. 1993, 51, 1.

31. Gundersen, P.; and Rasmussen, L.; Rev. Environ. Cont. Toxicol. 1990, 113, 1.

32. Armienta, M. A.; Rodrígues, R.; Queré, A.; Juaréz, F.; Cenciros, N.; and Aguayo, A.; Environ. Anal. Chem. 1993, 54,1 . 